\title{
Nonlinear instabilities of multi-site breathers in Klein-Gordon lattices
}

\author{
By Jesús Cuevas-Maraver, Panayotis G. Kevrekidis and Dmitry E. \\ Pelinovsky
}

In the present work, we explore the possibility of excited breather states in a nonlinear Klein-Gordon lattice to become nonlinearly unstable, even if they are found to be spectrally stable. The mechanism for this fundamentally nonlinear instability is through the resonance with the wave continuum of a multiple of an internal mode eigenfrequency in the linearization of excited breather states. For the nonlinear instability, the internal mode must have its Krein signature opposite to that of the wave continuum. This mechanism is not only theoretically proposed, but also numerically corroborated through two concrete examples of the KleinGordon lattice with a soft (Morse) and a hard $\left(\phi^{4}\right)$ potential. Compared to the case of the nonlinear Schrödinger lattice, the Krein signature of the internal mode relative to that of the wave continuum may change depending on the period of the excited breather state. For the periods for which the Krein signatures of the internal mode and the wave continuum coincide, excited breather states are observed to be nonlinearly stable.

\section{Introduction}

The study of anharmonic modes constructed out of a few excited lattices sites in nonlinear lattice dynamical systems is a broad and diverse theme of research that emerged in the physics literature through the work of [24, 34]. Subsequently, the mathematical proof of the existence of such modes in 20] not only placed such states on a rigorous mathematical footing, but also gave a deep set of insights towards their potentially generic nature. This, in turn, led to a considerable growth and diversification of interest into these modes over the last two decades, eventually culminating in a wide array of reviews on both the methods of analysis of these modes, as well as on their diverse applications [3, 13, 14, 19]. Noting only 
some among the many areas where these modes have been impacting, we cite coupled waveguide arrays and photorefractive crystals in the realm of nonlinear optics [6, 18, the denaturation dynamics of the DNA double strand in biophysics [30, breather formation in eletrical lattices and in micromechanical cantilever arrays [33], Bose-Einstein condensates in optical lattices in atomic physics [23], as well as bright and dark breathers in granular crystals [8, 10.

Orbital and asymptotic stability of the fundamental (single-site) breathers were established by Bambusi [4, 5. Spectral stability of more complicated multi-breathers were classified in the recent works [2, 17, 29], depending on the phase difference in the nonlinear oscillations between different sites of the lattice (and the nature of the potential). The simplest spectrally stable multi-breather configuration includes a two-site breather. If the two sites excited in the anti-continuum limit are adjacent in the lattice, then the spectrally stable breather is in-phase (anti-phase) for the hard (soft) potential $V$ [17, 29].

The main question we would like to consider is if the spectrally stable two-site breather is also stable in the nonlinear dynamics of the discrete Klein-Gordon (KG) equation (11). This is a part of the more general and broadly important question about whether spectrally stable excited (non-fundamental) states of a Hamiltonian system are nonlinearly stable or not. In a similar context of the discrete nonlinear Schrödinger (NLS) equation, the two-site breathers near the anti-continuum limit are not orbitally stable because the linearized spectrum admits an internal mode of negative energy (negative Krein signature) [26]. This internal mode of negative energy destabilizes nonlinear dynamics of the two-site breathers if a multiple harmonic of the internal mode eigenfrequency occurs in the frequency spectrum of the wave continuum that bears positive energy [11, 15.

In the context of the discrete KG equation (1), a similar concept of Krein signature can be introduced for the internal modes in the linearization of multi-site breather solutions [3, 21, 22]. The discrete NLS equation appears to be a valid approximation for the small-amplitude weakly coupled multi-site breathers [25]. Therefore, it is natural to expect that the mechanism of nonlinear instability based on the resonance between multiple harmonics of the internal mode eigenfrequency and the frequency spectrum of the wave continuum may also be observed for the two-site breathers near the anti-continuum limit. This conjecture is fully confirmed in this work. However, depending on the period of the limiting breather, one can find parameter configurations, where the nonlinear instability can be avoided, because the Krein signatures of both the internal mode and the wave continuum coincide. In such situations, our analytical 
and numerical results indicate that the two-site breathers are stable in the nonlinear dynamics of the discrete KG equation (1).

The presentation of our results is structured as follows. In section 2, we present the mathematical formalism of the problem. Upon setting up the Klein-Gordon lattices, their breather solutions and associated linearization (consisting of both internal modes and wave continuum), we explore the notion of their Krein signature. Subsequently, we use asymptotic expansions to reveal the nonlinear resonant mechanism leading to the main instability result of the present work. In section 3, we numerically corroborate the analytical results. We report computations of the Floquet spectrum associated with the breathers in two examples of the Klein-Gordon lattice associated with the Morse and hard $\phi^{4}$ potentials. We also present direct numerical simulations highlighting the outcomes of the unstable or stable dynamics of two-site breathers. Finally, in section 4 we summarize our results and present some directions for future work.

\section{Mathematical formalism}

We start with the one-dimensional Klein-Gordon (KG) lattice equation:

$$
\ddot{u}_{n}+V^{\prime}\left(u_{n}\right)=\varepsilon\left(u_{n+1}-2 u_{n}+u_{n-1}\right), \quad n \in \mathbb{Z},
$$

where $V: \mathbb{R} \rightarrow \mathbb{R}$ is an on-site (substrate) potential and $\varepsilon>0$ is the coupling constant. The amplitudes of coupled nonlinear oscillators on lattice sites form a sequence $\left\{u_{n}\right\}_{n \in \mathbb{Z}} \in \mathbb{R}^{\mathbb{Z}}$ denoted by the vector $\mathbf{u}$, which is typically defined in the sequence space $\ell^{2}(\mathbb{Z})$. The solution $\mathbf{u}$ of the discrete $\mathrm{KG}$ equation (11) is a function of time $t$. A local solution $\mathbf{u}$ exists in $C^{1}\left((-T, T) ; \ell^{2}(\mathbb{Z})\right)$ for some $T>0$ if $V^{\prime}$ is Lipschitz, thanks to the Picard's contraction method and the boundedness of the discrete Laplace operator in the lattice equation (11).

For numerical experiments, we consider two prototypical examples of smooth on-site potentials, namely the Morse and $\phi^{4}$ potentials, which are given, respectively, by

$$
\text { (i) } V(u)=\frac{1}{2}\left(e^{-u}-1\right)^{2} \text { and (ii) } V(u)=\frac{1}{2} u^{2}+\frac{1}{4} u^{4} \text {. }
$$

We note that $V(0)=V^{\prime}(0)=0$ and $V^{\prime \prime}(0)=1$ due to our normalization. The Morse potential (i) in (2) is classified as a soft potential because the period $T$ of oscillations in the nonlinear oscillator equation

$$
\ddot{\varphi}+V^{\prime}(\varphi)=0
$$

increases with the oscillator energy $E=\frac{1}{2} \dot{\varphi}^{2}+V(\varphi)\left[12\right.$. The $\phi^{4}$ potential (ii) in (2) is a hard potential because the period $T$ decreases with $E$. Fig. 

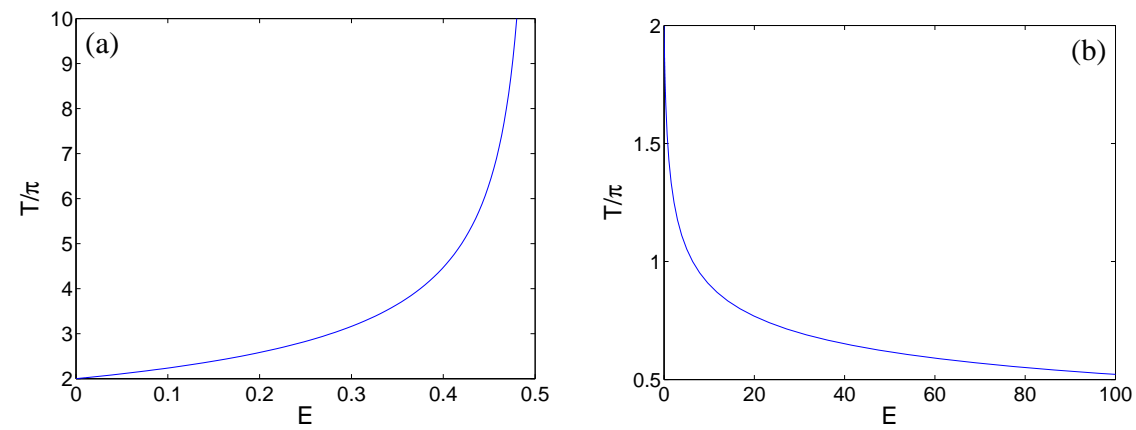

Figure 1. Dependence of the period $T$ with respect to the energy $E$ for the nonlinear oscillator (3) in the Morse (a) and $\phi^{4}$ (b) potentials.

1 shows the dependence of the period $T$ with respect to the energy $E$ for the Morse (a) and $\phi^{4}(\mathrm{~b})$ potentials.

The discrete KG equation (1D) is associated with the Hamiltonian

$$
H=\frac{1}{2} \sum_{n \in \mathbb{Z}} \dot{u}_{n}^{2}+\frac{\varepsilon}{2} \sum_{n \in \mathbb{Z}}\left(u_{n+1}-u_{n}\right)^{2}+\sum_{n \in \mathbb{Z}} V\left(u_{n}\right)
$$

The energy conservation (44) can be used to extend the local solution $\mathbf{u} \in$ $C^{1}\left((-T, T) ; \ell^{2}(\mathbb{Z})\right)$ to the global solution $\mathbf{u} \in C^{1}\left(\mathbb{R} ; \ell^{2}(\mathbb{Z})\right)$ if the potential $V$ possesses some coercivity. This is definitely the case for the hard $\phi^{4}$ potential (ii) in (2), which supports global solutions of the KG lattice equation (11) with time-independent bounds on $\|\mathbf{u}\|_{\ell^{2}}$ and $\|\dot{\mathbf{u}}\|_{\ell^{2}}$. Due to positivity of $V$ for the soft Morse potential (i), the local solution $\mathbf{u} \in$ $C^{1}\left((-T, T) ; \ell^{2}(\mathbb{Z})\right)$ is extended for $\varepsilon>0$ to the global solution with timeuniform bounds on $\|\dot{\mathbf{u}}\|_{\ell^{2}}$ and $\|\delta \mathbf{u}\|_{\ell^{2}}$, where $\delta$ is the difference operator defined by $(\delta \mathbf{u})_{n}=u_{n+1}-u_{n}, n \in \mathbb{Z}$. By using $\frac{d}{d t}\|\mathbf{u}\|_{\ell^{2}} \leq\|\dot{\mathbf{u}}\|_{\ell^{2}}$, we obtain a global but linearly growing bound on $\|\mathbf{u}\|_{\ell^{2}}$.

Let us mention in passing that the soft $\phi^{4}$ potential $V(u)=\frac{1}{2} u^{2}-\frac{1}{4} u^{4}$ supports the blow-up in a finite time for sufficiently large initial data [1]. To avoid the finite-time blow-up, we take the Morse potential $V$ in (i) as a representative of the class of soft potentials. The Morse potential has also applications in physics of DNA [31.

We consider the breather solutions of the discrete KG equation (1), which are $T$-periodic in time and exponentially localized in space. These solutions are given by the vector $\mathbf{u}(t)=\mathbf{u}(t+T)$ such that $\mathbf{u} \in$ $C_{\text {per }}^{\infty}\left((0, T) ; \ell^{2}(\mathbb{Z})\right)$ if $V$ is smooth. Breather solutions are constructed by the arguments based on the implicit function theorem for sufficiently 
small $\varepsilon>0$ [20] (see also review in [29]). In what follows, we always assume that the potential $V$ is smooth.

Adding a perturbation $\mathbf{w} \in C^{\infty}\left(\mathbb{R} ; \ell^{2}(\mathbb{Z})\right)$ to the breather solution $\mathbf{u}(t)=\mathbf{u}(t+T)$, where $\mathbf{u} \in C_{\mathrm{per}}^{\infty}\left((0, T) ; \ell^{2}(\mathbb{Z})\right)$, we obtain the linearized discrete $\mathrm{KG}$ equation in the form

$$
\ddot{w}_{n}+V^{\prime \prime}\left(u_{n}\right) w_{n}=\varepsilon\left(w_{n+1}-2 w_{n}+w_{n-1}\right), \quad n \in \mathbb{Z} .
$$

According to the Floquet theory, we are looking for solutions of the linearized equation (5) in the form $\mathbf{w}(t)=e^{\lambda t} \mathbf{W}(t)$, where $\lambda \in \mathbb{C}$ is a spectral parameter and $\mathbf{W} \in C_{\text {per }}^{\infty}\left((0, T) ; \ell^{2}(\mathbb{Z})\right)$ is the eigenvector of the spectral problem

$$
\ddot{W}_{n}+2 \lambda \dot{W}_{n}+\lambda^{2} W_{n}+V^{\prime \prime}\left(u_{n}\right) W_{n}=\varepsilon\left(W_{n+1}-2 W_{n}+W_{n-1}\right), \quad n \in \mathbb{Z} .
$$

Given an admissible value of the spectral parameter $\lambda$, we can compute the Floquet multiplier $\mu$ by $\mu=e^{\lambda T}$. The breather $\mathbf{u}$ is spectrally stable if all admissible values of the Floquet multipliers $\mu$ belong to the unit circle. Due to the reversibility and reality of the linearized discrete KG equation (5), the Floquet multipliers $\mu$ occur either in pairs on the unit circle $\{\mu, \bar{\mu}\}$ or real pairs $\left\{\mu, \mu^{-1}\right\}$ or quartets off the unit circle $\left\{\mu, \bar{\mu}, \mu^{-1}, \bar{\mu}^{-1}\right\}$. The breathers are spectrally unstable if real pairs or complex quartets exist outside the unit circle.

\subsection{Wave continuum}

The wave continuum is defined by solutions of the limiting spectral problem

$$
\ddot{W}_{n}+2 \lambda \dot{W}_{n}+\lambda^{2} W_{n}+W_{n}=\varepsilon\left(W_{n+1}-2 W_{n}+W_{n-1}\right), \quad n \in \mathbb{Z},
$$

which corresponds to the zero solution $\mathbf{u}=\mathbf{0}$. Performing the discrete Fourier transform,

$$
W_{n}(t)=\int_{-\pi}^{\pi} \hat{W}(t, \theta) e^{i n \theta} d \theta, \quad n \in \mathbb{Z},
$$

and using the Fourier series for the $T$-periodic functions

$$
\hat{W}(t, \theta)=\sum_{m \in \mathbb{Z}} \hat{W}_{m}(\theta) e^{i m \omega_{0} t}, \quad \omega_{0}=\frac{2 \pi}{T},
$$

we obtain the dispersion equation for the spectral bands

$$
\lambda=i\left( \pm \omega(\theta)-m \omega_{0}\right), \quad m \in \mathbb{Z},
$$


where the function $\omega:[-\pi, \pi] \rightarrow[1, \sqrt{1+4 \varepsilon}]$ represents the fundamental band of the wave continuum given by

$$
\omega(\theta):=\sqrt{1+4 \varepsilon \sin ^{2}\left(\frac{\theta}{2}\right)}, \quad \theta \in[-\pi, \pi] .
$$

In terms of the Floquet multiplier $\mu=e^{\lambda T}$, only two spectral bands show up symmetrically on the unit circle. The wave continuum corresponds to the Floquet multipliers at $\mu=e^{ \pm i \omega(\theta) T}, \theta \in[-\pi, \pi]$. The two spectral bands shrink to the two points of infinite multiplicities at $\mu=e^{ \pm i T}$ in the anti-continuum limit $\varepsilon \rightarrow 0$.

\subsection{Krein signature}

The Krein quantity represents the symplectic structure of the linearized discrete KG equation (5). It is used to characterize Floquet multipliers on the unit circle and stability transitions of discrete breathers [3, 21, 22. Recently, the same quantity is used in the context of stability of the periodic travelling waves in the FPU lattices [7]. Using variables $\left\{w_{n}, p_{n}\right\}_{n \in \mathbb{Z}}$, we can write (5) in the form,

$$
\frac{d w_{n}}{d t}=\frac{\partial \mathcal{H}}{\partial p_{n}}, \quad \frac{d p_{n}}{d t}=-\frac{\partial \mathcal{H}}{\partial w_{n}}, \quad n \in \mathbb{Z},
$$

where $\mathcal{H}$ is the second variation of the Hamiltonian $H$ in (4) given by

$$
\mathcal{H}=\frac{1}{2} \sum_{n \in \mathbb{Z}} p_{n}^{2}+\frac{\varepsilon}{2} \sum_{n \in \mathbb{Z}}\left(w_{n+1}-w_{n}\right)^{2}+\frac{1}{2} \sum_{n \in \mathbb{Z}} V^{\prime \prime}\left(u_{n}\right) w_{n}^{2} .
$$

Then, the symplectic (Krein) quantity is given for any $\mathbf{w}, \mathbf{p} \in \ell^{2}(\mathbb{Z})$ by

$$
K=i \sum_{n \in \mathbb{Z}}\left(\bar{p}_{n} w_{n}-p_{n} \bar{w}_{n}\right)
$$

We note that $K$ is real and constant in time $t$. It is identically zero at the real-valued solutions of the linearized discrete KG equation (5). However, it is nonzero for complex-valued solutions, e.g. for eigenfunctions $\mathbf{w}(t)=$ $e^{i \Omega t} \mathbf{W}(t)$, where $\lambda=i \Omega$ and $\mathbf{W} \in C_{\text {per }}^{\infty}\left((0, T) ; \ell^{2}(\mathbb{Z})\right)$ are solutions of the spectral problem (6). In this case, the Krein quantity $K$ can be written in the equivalent form

$$
K=2 \Omega \sum_{n \in \mathbb{Z}}\left|W_{n}\right|^{2}+i \sum_{n \in \mathbb{Z}}\left(\dot{\bar{W}}_{n} W_{n}-\dot{W}_{n} \bar{W}_{n}\right) .
$$

For the wave continuum, given by the Fourier transform (8) and the Fourier series (9) for $\Omega= \pm \omega(\theta)-m \omega_{0}$ with $\theta \in[-\pi, \pi]$ and $m \in \mathbb{Z}$, we 
obtain

$$
K= \pm 4 \pi \int_{-\pi}^{\pi} \omega(\theta)\left|\hat{W}_{m}(\theta)\right|^{2} d \theta .
$$

Since $\omega(\theta)>0$ for every $\theta \in[-\pi, \pi]$, according to the dispersion relation (11), we have $K>0$ for each $m$-th spectral band with $\Omega=\omega(\theta)-m \omega_{0}$ and $K<0$ for each $m$-th spectral band with $\Omega=-\omega(\theta)-m \omega_{0}$, for every $m \in \mathbb{Z}$.

\subsection{Internal modes of multi-site breathers}

The internal modes correspond to isolated Floquet multipliers on the unit circle that occur commonly in the linearization of multi-site breathers [16. 27. The internal modes and their Krein signature are well approximated near the anti-continuum limit of weak coupling between the nonlinear oscillators. Asymptotic expansions for eigenvalues and eigenfunctions of the spectral problem (6) for small $\varepsilon$ are reported in the most general case in [29]. Here we review these expansions in the particular case, when the nonlinear oscillators are excited on adjacent lattice sites as $\varepsilon \rightarrow 0$ (see also [12, 17]).

Let us represent the multi-site breather by its limiting configuration

$$
\mathbf{u}_{\lim }(t):=\lim _{\varepsilon \rightarrow 0} \mathbf{u}(t)=\sum_{j=1}^{N} \varphi\left(t+\left(\sigma_{j}-1\right) T / 4\right) \mathbf{e}_{j},
$$

where $N$ is the number of excited oscillators, $\mathbf{e}_{j}$ is the unit vector supported at the $j$-th lattice node, $\sigma_{j} \in\{+1,-1\}$ for $j=1,2, \ldots, N$, and $\varphi \in C_{\text {per }}^{\infty}(0, T)$ is a unique, even $T$-periodic solution of the nonlinear oscillator equation (3) such that $\varphi(0)>0$. The two nonlinear oscillators at the $j$-th and $(j+1)$-th lattice nodes are in phase if $\sigma_{j} \sigma_{j+1}=1$ and anti-phase if $\sigma_{j} \sigma_{j+1}=-1$. Under this choice of in-phase and anti-phase configurations, it is proved that the multi-site breather solution $\mathbf{u} \in C_{\text {per }}^{\infty}\left((0, T) ; \ell^{2}(\mathbb{Z})\right)$ is uniquely continued from its limit (16) for small $\varepsilon>0$ and the correction term $\mathbf{u}-\mathbf{u}_{\text {lim }}$ is $\mathcal{O}_{\ell^{2}}(\varepsilon)$ [20, 29].

The spectral stability problem (6) with $\varepsilon=0$ associated with the limiting breather (16) has the Floquet multiplier $\mu=1$ of algebraic multiplicity $2 N$ and the pair of Floquet multipliers $\mu=e^{ \pm i T}$ of infinite algebraic multiplicities. The two spectral bands of the wave continuum on the unit circle bifurcate from the pair of Floquet multipliers $\mu=e^{ \pm i T}$. The Krein signature of the two spectral bands is $\operatorname{sign}(K)= \pm 1$. Isolated Floquet multipliers may also bifurcate from the pair of Floquet multipliers $\mu=e^{ \pm i T}$ to share the same Krein signature as the one for the spectral band they bifurcate from. In the context of the discrete NLS equation, the absence of isolated Floquet multipliers bifurcating from the spectral 
bands can be proved under some restrictive conditions 28. In either case, our main interest is to study internal modes arising as a result of splitting of the Floquet multiplier $\mu=1$ of algebraic multiplicity $2 N$ under the perturbation terms with small $\varepsilon$.

Representing the small spectral parameter $\lambda=\varepsilon^{\frac{1}{2}} \Lambda$ in the spectral problem (6), the authors of [29] justified the following asymptotic expansion for the eigenvector $\mathbf{W}$ of the spectral problem (6):

$\mathbf{W}(t)=\sum_{j=1}^{N} c_{j} \dot{\varphi}\left(t+\left(\sigma_{j}-1\right) T / 4\right) \mathbf{e}_{j}-\varepsilon^{\frac{1}{2}} \Lambda \sum_{j=1}^{N} c_{j} v\left(t+\left(\sigma_{j}-1\right) T / 4\right) \mathbf{e}_{j}+\varepsilon \mathbf{W}_{\mathrm{rem}}(t)$,

where the even $T$-periodic function $v \in C_{\text {per }}^{\infty}(0, T)$ is uniquely determined by the solution of the inhomogeneous equation

$$
\ddot{v}+V^{\prime \prime}(\varphi) v=2 \ddot{\varphi}
$$

$\mathbf{c}=\left(c_{1}, c_{2}, \ldots, c_{N}\right)$ is the vector of projections to be determined, $\Lambda$ is the new spectral parameter, and $\mathbf{W}_{\text {rem }}$ is the remainder term of the asymptotic expansion. The values of $\sigma_{j} \in\{+1,-1\}$ for $j=1,2, \ldots, N$ are the same as in (16).

In the case of symmetric potentials $V$ satisfying $V(-u)=V(u)$, it is shown in [29] that $\mathbf{W}_{\text {rem }} \in C_{\text {per }}^{\infty}\left((0, T) ; \ell^{2}(\mathbb{Z})\right)$ exists only if the spectral parameter $\Lambda$ is defined by the matrix eigenvalue problem for the eigenvector c:

$$
-\frac{T(E)^{2}}{S T^{\prime}(E)} \Lambda^{2} \mathbf{c}=M \mathbf{c}
$$

where $S=\int_{0}^{T} \dot{\varphi}^{2}(t) d t$ is a positive constant coefficient, $T^{\prime}(E)$ is the derivative of the period of the nonlinear oscillator equation (3) with respect to its energy $E=\frac{1}{2} \dot{\varphi}^{2}+V(\varphi)$, see Fig. 1, and $M$ is the tri-diagonal matrix given by the elements

$$
M_{i j}=\left\{\begin{array}{cc}
-\sigma_{j}\left(\sigma_{j+1}+\sigma_{j-1}\right), & i=j, \\
1, & i=j \pm 1,
\end{array}\right.
$$

subject to the Dirichlet boundary conditions. In the case of hard potential with $T^{\prime}(E)<0$ and for the two-site in-phase breather with $N=2$ and $\sigma_{1}=\sigma_{2}$, the matrix eigenvalue problem (19) has the double zero eigenvalue $\Lambda=0$ and a pair of purely imaginary eigenvalues

$$
\Lambda= \pm i \Omega_{0}, \quad \Omega_{0}=\frac{\sqrt{2 S\left|T^{\prime}(E)\right|}}{T(E)}>0
$$

The same conclusion holds for the soft potential with $T^{\prime}(E)>0$ but applies to the two-site anti-phase breather with $N=2$ and $\sigma_{1}=-\sigma_{2}$. 
The asymptotic approximation (201) corresponds to the pair of Floquet multipliers on the unit circle, which are given at the leading order by $\mu=e^{ \pm i \varepsilon^{\frac{1}{2}} \Omega_{0}}$.

We shall now substitute the asymptotic expansion (17) with $\Lambda=i \Omega_{0}$ into the Krein quantity (15) and compute the Krein signature of the internal modes (20) to the leading order in $\varepsilon$. After straightforward computations, we obtain $K=\varepsilon^{\frac{1}{2}} K_{1}+\mathcal{O}(\varepsilon)$, where

$$
K_{1}=2 \Omega_{0} \sum_{j=1}^{N} A\left|c_{j}\right|^{2}, \quad A:=\dot{\varphi}^{2}-\dot{\varphi} \dot{v}+v \ddot{\varphi} .
$$

By the time-conservation of $K$, it is clear that $A$ is independent of $t$. Let us compute this quantity explicitly by using the exact expression for $v$ satisfying the linear inhomogeneous equation (18) obtained in [29]:

$$
v(t)=t \dot{\varphi}(t)+\frac{T(E)}{T^{\prime}(E)} \partial_{E} \varphi(t ; E),
$$

where $\varphi(t ; E)$ denotes the family of even $T(E)$-periodic solutions of the nonlinear oscillator equation (3) satisfying the constraint $\varphi(0 ; E)=$ $a(E)>0$, where $a(E)$ is the first positive root of $V(a(E))=E$. Substituting $v$ into the formula for $A$, we obtain

$$
A=\frac{T(E)}{T^{\prime}(E)}\left[\ddot{\varphi}(t) \partial_{E} \varphi(t ; E)-\dot{\varphi} \partial_{E} \dot{\varphi}(t ; E)\right]=-\frac{T(E)}{T^{\prime}(E)},
$$

where the last identity holds because the time-independent Wronskian of two linearly independent solutions of the homogeneous equation $\ddot{u}+$ $V^{\prime \prime}(\varphi) u=0$ is given explicitly by

$$
\begin{aligned}
W & :=\ddot{\varphi}(t) \partial_{E} \varphi(t ; E)-\dot{\varphi} \partial_{E} \dot{\varphi}(t ; E)=\ddot{\varphi}(0) \partial_{E} \varphi(0 ; E)-\dot{\varphi} \partial_{E} \dot{\varphi}(0 ; E) \\
& =\ddot{\varphi}(0) a^{\prime}(E)=-V^{\prime}(a(E)) a^{\prime}(E)=-1 .
\end{aligned}
$$

Substituting (22) into (21), we obtain

$$
K=-\frac{2 \Omega_{0} T(E)}{T^{\prime}(E)} \varepsilon^{\frac{1}{2}} \sum_{j=1}^{N}\left|c_{j}\right|^{2}+\mathcal{O}(\varepsilon) .
$$

For the eigenvalue $\lambda=i \varepsilon^{\frac{1}{2}} \Omega_{0}+\mathcal{O}(\varepsilon)$ with $\Omega_{0}$ given by (20) associated with the the internal mode of the two-site breather, we have $K>0$ for the hard potential with $T^{\prime}(E)<0$ and $K<0$ for the soft potential with $T^{\prime}(E)<0$.

Recall that $T(E) \rightarrow 2 \pi$ as $E \rightarrow 0$, where $E \rightarrow 0$ represents the smallamplitude limit in the nonlinear oscillator equation (3). We assume that $T^{\prime}(E)$ remains sign-definite for all admissible values of the energy pa- 
rameter $E$. Let us now summarize on the Krein signatures for the wave continuum and the internal mode of the two-site breathers:

- For the hard potential with $T^{\prime}(E)<0$, the internal mode with the Floquet multiplier $\mu=e^{i T\left(\varepsilon^{\frac{1}{2}} \Omega_{0}+\mathcal{O}(\varepsilon)\right)}$ has the positive Krein signature and so is the Krein signature for the wave spectrum associated with the spectral band of Floquet multipliers $\mu=e^{i T(1+\mathcal{O}(\varepsilon))}$. Since $T(E)<2 \pi$ for the hard potentials, we have two situations:

$-0<T<\pi$ : the Krein signatures of the internal mode and the wave spectrum in the upper semi-circle coincide;

$-\pi<T<2 \pi$ : the Krein signatures of the internal mode and the wave spectrum in the upper semi-circle are opposite to each other.

- For the soft potential with $T^{\prime}(E)>0$, the internal mode with the Floquet multiplier $\mu=e^{i T\left(\varepsilon^{\frac{1}{2}} \Omega_{0}+\mathcal{O}(\varepsilon)\right)}$ has the negative Krein signature, which is opposite to the Krein signature for the wave spectrum corresponding to the spectral band of Floquet multipliers $\mu=e^{i T(1+\mathcal{O}(\varepsilon))}$. Since $T(E)>2 \pi$ for the soft potentials, we shall restrict out attention to the interval of $T$ between $2 \pi$ and $4 \pi$ (which is the $1: 2$ resonance). If $2 \pi<T<4 \pi$, we have two situations:

$-2 \pi<T<3 \pi$ : the Krein signatures of the internal mode and the wave spectrum in the upper semi-circle are opposite to each other;

$-3 \pi<T<4 \pi$ : the Krein signatures of the internal mode and the wave spectrum in the upper semi-circle coincide.

We will show that the nonlinear instability occurs when the Krein signatures of the internal mode and the wave spectrum are opposite to each other. At the same time, the two-site breathers are nonlinearly stable if the two signatures coincide.

\subsection{Asymptotic expansions}

We describe now the main result of this work. We show with the use of formal asymptotic expansions that the multi-site breathers are unstable in the discrete KG equation (11) if the Krein signatures of the internal mode and the wave spectrum are opposite to each other. To study the nonlinear dynamics of the internal mode, we adopt the asymptotic expansions obtained earlier for the continuous and discrete NLS models [15, 27]. We assume the following spectral properties:

P1 There exists a unique internal mode with eigenfrequency $\Omega$ such that the Floquet multiplier $\mu=e^{i \Omega T}$ is isolated from the spectral bands 
located at $\mu=e^{ \pm i \omega(\theta) T}, \theta \in[-\pi, \pi]$, where $\omega(\theta)$ is given by (11). To be mathematically precise, we assume that

$$
\Omega \in\left(0, \min \left\{1-k_{0} \omega_{0}, m_{0} \omega_{0}-\sqrt{1+4 \varepsilon}\right\}\right),
$$

where $\omega_{0}=\frac{2 \pi}{T}$ is the breather frequency, $k_{0} \in \mathbb{N}$ is the maximal integer such that $1-k_{0} \omega_{0}>0$, whereas $m_{0} \in \mathbb{N}$ is the minimal integer such that $m_{0} \omega_{0}-\sqrt{1+4 \varepsilon}>0$.

P2 The double frequency of the internal mode eigenfrequency $\Omega$ belongs to the spectral band of the wave spectrum, that is,

either $2 \Omega \in\left(1-k_{0} \omega_{0}, \sqrt{1+4 \varepsilon}-k_{0} \omega_{0}\right) \quad$ or $\quad 2 \Omega \in\left(m_{0} \omega_{0}-\sqrt{1+4 \varepsilon}, m_{0} \omega_{0}-1\right)$,

where $\omega_{0}, k_{0}$, and $m_{0}$ are the same as for (25).

P3 There exists a unique eigenvector $\dot{\mathbf{u}} \in C_{\mathrm{per}}^{\infty}\left((0, T) ; \ell^{2}(\mathbb{Z})\right)$ of the spectral problem (6) with $\lambda=0$, where $\mathbf{u} \in C_{\mathrm{per}}^{\infty}\left((0, T) ; \ell^{2}(\mathbb{Z})\right)$ is the breather of the discrete KG equation (1).

P4 The $T$-periodic functions $\dot{u}_{n}$ and $V^{\prime \prime \prime}\left(u_{n}\right)\left|W_{n}\right|^{2}$ are respectively odd and even in the time variable $t$ for every $n \in \mathbb{Z}$, where $\mathbf{W} \in$ $C_{\text {per }}^{\infty}\left((0, T) ; \ell^{2}(\mathbb{Z})\right)$ is the internal mode for the eigenvalue $\lambda=i \Omega$ found from the spectral problem (6).

We introduce a small parameter $\delta$ that stands for the amplitude of the internal mode. The small parameter $\delta$ is unrelated with the possibly small parameter $\varepsilon$. Then, we look for an asymptotic expansion

$$
\mathbf{U}(t)=\mathbf{u}(t)+\delta \mathbf{u}^{(1)}(t)+\delta^{2} \mathbf{u}^{(2)}(t)+\delta^{3} \mathbf{u}^{(3)}(t)+\cdots,
$$

where $\mathbf{u} \in C_{\text {per }}^{\infty}\left((0, T) ; \ell^{2}(\mathbb{Z})\right)$ is the underlying breather. We choose the first-order correction term in the form,

$$
\mathbf{u}^{(1)}(t)=c(\tau) \mathbf{W}(t) e^{i \Omega t}+\bar{c}(\tau) \overline{\mathbf{W}}(t) e^{-i \Omega t}, \quad \tau=\delta^{2} t,
$$

where $c$ is the slowly varying amplitude, $\mathbf{W} \in C_{\text {per }}^{\infty}\left((0, T) ; \ell^{2}(\mathbb{Z})\right)$ is the eigenvector of the spectral problem (6) for the eigenvalue $\lambda=i \Omega$ isolated from the wave spectrum, see property (P1).

Separating the variables for the second-order correction term, we represent

$$
\mathbf{u}^{(2)}(t)=c(\tau)^{2} \mathbf{P}(t) e^{2 i \Omega t}+|c(\tau)|^{2} \mathbf{Q}(t)+\bar{c}(\tau)^{2} \overline{\mathbf{P}}(t) e^{-2 i \Omega t},
$$

where $\mathbf{P}, \mathbf{Q} \in C_{\text {per }}^{\infty}\left((0, T) ; \ell^{\infty}(\mathbb{Z})\right)$ are solutions of the linear inhomogeneous equations

$\ddot{P}_{n}+4 i \Omega \dot{P}_{n}-4 \Omega^{2} P_{n}+V^{\prime \prime}\left(u_{n}\right) P_{n}=\varepsilon\left(P_{n+1}-2 P_{n}+P_{n-1}\right)-\frac{1}{2} V^{\prime \prime \prime}\left(u_{n}\right) W_{n}^{2}, \quad n \in \mathbb{Z}$ 
and

$$
\ddot{Q}_{n}+V^{\prime \prime}\left(u_{n}\right) Q_{n}=\varepsilon\left(Q_{n+1}-2 Q_{n}+Q_{n-1}\right)-V^{\prime \prime \prime}\left(u_{n}\right)\left|W_{n}\right|^{2}, \quad n \in \mathbb{Z} .
$$

Although the linear operator in the linear equation (31) is not invertible because $\dot{\mathbf{u}} \in C_{\mathrm{per}}^{\infty}\left((0, T) ; \ell^{2}(\mathbb{Z})\right)$ is a solution of the homogeneous equation, see property (P3), the Fredholm solvability condition is satisfied

$$
\sum_{n \in \mathbb{Z}} \int_{0}^{T} V^{\prime \prime \prime}\left(u_{n}\right)\left|W_{n}\right|^{2} \dot{u}_{n} d t=0,
$$

because $\dot{u}_{n}$ is odd $T$-periodic and $V^{\prime \prime \prime}\left(u_{n}\right)\left|W_{n}\right|^{2}$ is even $T$-periodic functions with respect to the time variable $t$ for every $n \in \mathbb{Z}$, see property (P4). Consequently, there is a unique even $T$-periodic solution $\mathbf{Q} \in C_{\text {per }}^{\infty}\left((0, T) ; \ell^{2}(\mathbb{Z})\right)$ of the linear inhomogeneous equation (31).

The linear operator in the linear equation (30) is not invertible because $2 \Omega$ belongs to the spectral band of the wave spectrum, see property (P2). Consequently, the bounded even $T$-periodic solution $\mathbf{P} \in$ $C_{\text {per }}^{\infty}\left((0, T) ; \ell^{\infty}(\mathbb{Z})\right)$ is not uniquely defined unless the boundary conditions are added as $n \rightarrow \pm \infty$. To obtain a unique bounded solution for $\mathbf{P}$, we specify the Sommerfeld radiation boundary conditions as follows.

Let $\sigma=1$ if $T \in(0, \pi) \bmod (2 \pi)$ and $\sigma=-1$ if $T \in$ $(\pi, 2 \pi) \bmod (2 \pi)$. Then, we require that the even $T$-periodic solution $\mathbf{P} \in C_{\text {per }}^{\infty}\left((0, T) ; \ell^{\infty}(\mathbb{Z})\right)$ of the linear inhomogeneous equation (30) satisfies the boundary conditions

$$
R_{ \pm}(t)=\lim _{n \rightarrow \pm \infty} P_{n}(t) e^{ \pm i \sigma n \theta_{0}}
$$

where $R_{ \pm} \in C_{\text {per }}^{\infty}(0, T)$ are uniquely defined and $\theta_{0} \in(0, \pi)$ is uniquely found from the solution of the transcendental equations:

$$
\sigma=1: \quad \omega\left(\theta_{0}\right)-k_{0} \omega_{0}=2 \Omega \quad \text { or } \quad \sigma=-1: \quad m_{0} \omega_{0}-\omega\left(\theta_{0}\right)=2 \Omega,
$$

where $\omega(\theta)$ is given by the dispersion relation (11), $\omega_{0}=\frac{2 \pi}{T}$ is the breather frequency, $k_{0} \in \mathbb{N}$ is the maximal integer such that $1-k_{0} \omega_{0}>0$, whereas $m_{0} \in \mathbb{N}$ is the minimal integer such that $m_{0} \omega_{0}-\sqrt{1+4 \varepsilon}>0$. The existence of a unique $\theta_{0} \in(0, \pi)$ follows from the assumption (P2). We assume (and this will be a subject of the forthcoming work) that there exists a unique even $\mathbf{P} \in C_{\text {per }}^{\infty}\left((0, T) ; \ell^{\infty}(\mathbb{Z})\right)$ satisfying (30) and (32).

The Sommerfeld radiation boundary conditions (32) have the following physical meaning. If $\sigma=1$ and $\varepsilon$ is sufficiently small, the spectral band for the wave spectrum corresponding to the Floquet multipliers $\mu=e^{i \omega(\theta) T}$, $\theta \in[-\pi, \pi]$ is located in the upper semi-circle. Then, as $n \rightarrow \pm \infty$, the asymptotic solution (27) with the radiation boundary conditions (32) 
becomes

$U_{n}(t) \sim \delta^{2}\left(R_{ \pm}(t) c^{2}(\tau) e^{\mp i n \theta_{0}+i\left(\omega\left(\theta_{0}\right)-k_{0} \omega_{0}\right) t}+\bar{R}_{ \pm}(t) \bar{c}^{2}(\tau) e^{ \pm i n \theta_{0}-i\left(\omega\left(\theta_{0}\right)-k_{0} \omega_{0}\right) t}\right)+\mathcal{O}\left(\delta^{3}\right)$.

Recall that $\theta_{0} \in(0, \pi)$ and $\omega^{\prime}\left(\theta_{0}\right)>0$, where the derivative of $\omega$ in $\theta$ determines the group velocity of the linear wave packets. Therefore, the linear wave packets far from the breather field propagate outwards from $n=0$, where the breather is localized. On the other hand, if $\sigma=-1$, the spectral band for the wave spectrum corresponding to the Floquet multipliers $\mu=e^{i \omega(\theta) T}, \theta \in[-\pi, \pi]$ is now located in the lower semicircle, so that the Floquet multiplier $\mu=e^{2 i \Omega}$ for the double frequency $2 \Omega$ is in resonance with the spectral band corresponding to the Floquet multipliers $\mu=e^{-i \omega(\theta) T}, \theta \in[-\pi, \pi]$. Then, as $n \rightarrow \pm \infty$, the asymptotic solution (27) with radiation boundary conditions (32) becomes

$U_{n}(t) \sim \delta^{2}\left(R_{ \pm}(t) c^{2}(\tau) e^{ \pm i n \theta_{0}+i\left(m_{0} \omega_{0}-\omega\left(\theta_{0}\right)\right) t}+\bar{R}_{ \pm}(t) \bar{c}^{2}(\tau) e^{\mp i n \theta_{0}-i\left(m_{0} \omega_{0}-\omega\left(\theta_{0}\right)\right) t}\right)+\mathcal{O}\left(\delta^{3}\right)$.

After the change in the sign of the Sommerfeld boundary conditions (32), the linear wave packets far from the breather field still propagate outwards from $n=0$, where the breather is localized.

Finally, separating the variables for the third-order correction term, we represent

$$
\mathbf{u}^{(3)}(t)=c(\tau)^{3} \mathbf{G}(t) e^{3 i \Omega t}+\mathbf{F}(t, \tau) e^{i \Omega t}+\overline{\mathbf{F}}(t, \tau) e^{-i \Omega t}+\bar{c}(\tau)^{3} \overline{\mathbf{G}}(t) e^{-3 i \Omega t},
$$

where $\mathbf{F}(\cdot, \tau), \mathbf{G} \in C_{\text {per }}^{\infty}\left((0, T) ; \ell^{\infty}(\mathbb{Z})\right)$ are solutions of the linear inhomogeneous equations for every $\tau$. We only write the problem for $\mathbf{F}$, because it yields a solvability condition on the slowly varying amplitude $c$ of the internal mode. The function $\mathbf{F}$ is found from the linear inhomogeneous equation:

$$
\ddot{F}_{n}+2 i \Omega \dot{F}_{n}-\Omega^{2} F_{n}+V^{\prime \prime}\left(u_{n}\right) F_{n}=\varepsilon\left(F_{n+1}-2 F_{n}+F_{n-1}\right)+H_{n}, \quad n \in \mathbb{Z}
$$

where the dots denote derivatives with respect to $t$ and the source term $\mathbf{H}$ is given by

$H_{n}=-2 \dot{c}\left(\dot{W}_{n}+i \Omega W_{n}\right)-|c|^{2} c V^{\prime \prime \prime}\left(u_{n}\right)\left(W_{n} Q_{n}+\bar{W}_{n} P_{n}\right)-\frac{1}{2}|c|^{2} c V^{\prime \prime \prime \prime}\left(u_{n}\right)\left|W_{n}\right|^{2} W_{n}, \quad n \in \mathbb{Z}$.

The linear operator in the linear equation (35) is not invertible because $\mathbf{W} \in C_{\text {per }}^{\infty}\left((0, T) ; \ell^{2}(\mathbb{Z})\right)$ is a homogeneous solution, see property $(\mathrm{P} 1)$. Therefore, a solution exists for $\mathbf{F}(\cdot, \tau) \in C_{\text {per }}^{\infty}\left((0, T) ; \ell^{2}(\mathbb{Z})\right)$ if and only if the source term $\mathbf{H}$ satisfies the Fredholm solvability condition

$$
\frac{1}{T} \sum_{n \in \mathbb{Z}} \int_{0}^{T} \bar{W}_{n}(t) H_{n}(t, \tau) d t=0
$$


which yields the amplitude equation

$$
i K \frac{d c}{d \tau}+\beta|c|^{2} c=0,
$$

where $K$ is the same as the time-independent Krein quantity (15),

$K=-\frac{2 i}{T} \sum_{n \in \mathbb{Z}} \int_{0}^{T} \bar{W}_{n}\left(\dot{W}_{n}+i \Omega W_{n}\right) d t=2 \Omega \sum_{n \in \mathbb{Z}}\left|W_{n}\right|^{2}+i \sum_{n \in \mathbb{Z}}\left(\dot{\bar{W}}_{n} W_{n}-\dot{W}_{n} \bar{W}_{n}\right)$,

whereas $\beta$ is the coefficient of the cubic term given by

$$
\beta=\frac{1}{T} \sum_{n \in \mathbb{Z}} \int_{0}^{T}\left(V^{\prime \prime \prime}\left(u_{n}\right)\left(\left|W_{n}\right|^{2} Q_{n}+\bar{W}_{n}^{2} P_{n}\right)+\frac{1}{2} V^{\prime \prime \prime \prime}\left(u_{n}\right)\left|W_{n}\right|^{4}\right) d t .
$$

The coefficient $\beta$ is complex because the bounded but non-decaying vector $\mathbf{P}$ is complex-valued, according to the radiation boundary conditions (32). We are only interested in the imaginary part of $\beta$, which can be computed by using the linear inhomogeneous equation (30) and integration by parts:

$$
\begin{aligned}
2 i \operatorname{Im}(\beta) & =\frac{1}{T} \sum_{n \in \mathbb{Z}} \int_{0}^{T} V^{\prime \prime \prime}\left(u_{n}\right)\left(\bar{W}_{n}^{2} P_{n}-W_{n}^{2} \bar{P}_{n}\right) d t \\
& =\frac{2}{T} \sum_{n \in \mathbb{Z}} \int_{0}^{T}\left[\bar{P}_{n}\left(\ddot{P}_{n}+4 i \Omega \dot{P}_{n}-\varepsilon(\Delta P)_{n}\right)-P_{n}\left(\ddot{\bar{P}}_{n}-4 i \Omega \dot{\bar{P}}_{n}-\varepsilon(\Delta \bar{P})_{n}\right)\right] d t \\
& =\frac{2 \varepsilon}{T} \sum_{n \in \mathbb{Z}} \int_{0}^{T}\left[P_{n}(\Delta \bar{P})_{n}-\bar{P}_{n}(\Delta P)_{n}\right] d t
\end{aligned}
$$

where we have used notation $(\Delta P)_{n}=P_{n+1}-2 P_{n}+P_{n-1}$ for the discrete Laplacian operator. Note that the integration by parts in $t$ yields a vanishing result because $\mathbf{P} \in C_{\text {per }}^{\infty}\left((0, T) ; \ell^{\infty}(\mathbb{Z})\right)$. However, because $P_{n}$ does not decay to zero as $|n| \rightarrow \infty$, we have a nonzero result for $\operatorname{Im}(\beta)$. Indeed, we can write

$P_{n}(\Delta \bar{P})_{n}-\bar{P}_{n}(\Delta P)_{n}=S_{n}-S_{n-1}, \quad S_{n}:=P_{n}\left(\bar{P}_{n+1}-\bar{P}_{n}\right)-\bar{P}_{n}\left(P_{n+1}-P_{n}\right), \quad n \in \mathbb{Z}$,

interchange the integration and summation, and use the telescopic summations to obtain

$$
2 i \operatorname{Im}(\beta)=\frac{2 \varepsilon}{T} \int_{0}^{T}\left[\lim _{n \rightarrow+\infty} S_{n}-\lim _{n \rightarrow-\infty} S_{n}\right] d t .
$$

Substituting the Sommerfeld boundary conditions (32), we arrive to the sign-definite expression,

$$
2 i \operatorname{Im}(\beta)=4 \varepsilon \sigma \sin \left(\theta_{0}\right) \frac{1}{T} \int_{0}^{T}\left(\left|R_{+}(t)\right|^{2}+\left|R_{-}(t)\right|^{2}\right) d t
$$


where $\sin \left(\theta_{0}\right)>0$ for $\theta_{0} \in(0, \pi)$. It follows that $\operatorname{Im}(\beta) \neq 0$, if the radiation amplitudes $R_{ \pm}$in the boundary conditions (32) are nonzero. Multiplying (37) by $\bar{c}$ and subtracting the complex conjugate equation, we obtain the rate of change for the squared amplitude:

$$
K \frac{d|c|^{2}}{d \tau}=-4 \varepsilon \sigma \sin \left(\theta_{0}\right)|c|^{4} \frac{1}{T} \int_{0}^{T}\left(\left|R_{+}(t)\right|^{2}+\left|R_{-}(t)\right|^{2}\right) d t
$$

It follows from this equation that the squared amplitude $|c|^{2}$ decays to zero if $\operatorname{sign}(K)=\operatorname{sign}(\sigma)$ and grows if $\operatorname{sign}(K)=-\operatorname{sign}(\sigma)$. This is the main result of the asymptotic theory. In the former case, we can anticipate that the multi-site breathers are stable in the nonlinear dynamics of the discrete KG equation. In the latter case, we can predict that the multi-site breathers are nonlinearly unstable, in spite of their linearized stability.

In the approximation of the small-amplitude breathers with the discrete NLS equations [25], $T$ is close to $2 \pi$, so both hard and soft potentials feature nonlinear instability of the two-site breathers. This phenomenon was discovered recently for the discrete NLS equation in [15], following upon the earlier abstract analysis of the continuous NLS equation in [11]. Note that the conclusion changes drastically when the breather period is either smaller than $\pi$ or bigger than $3 \pi$. The former case is observed for small-period breathers in the hard potentials. The latter case is observed for breathers in the soft potentials near the $1: 2$ resonance. In either case, the multi-site breathers are expected to be nonlinearly stable.

\section{Numerical results}

Having explored the fundamentals of the nonlinear instability of multisite breathers, we now turn to a numerical examination of the discrete KG equation (11) for two potentials in (2), namely the soft (Morse) and the hard $\left(\phi^{4}\right)$ potentials. We choose two-site breathers with the excited sites at $n=0$ and $n=1$ oscillating in anti-phase for soft potentials, so that $u_{0}(0)=-u_{1}(0)$, and oscillating in phase for hard potentials, so that $u_{0}(0)=u_{1}(0)$. According to section 2.3, these two-site breathers

possess an internal mode with the small eigenfrequency $\Omega=\varepsilon^{\frac{1}{2}} \Omega_{0}+\mathcal{O}(\varepsilon)$, where $\Omega_{0}$ is given by (20). The corresponding pair of Floquet multipliers $\mu=e^{ \pm i \Omega T}$ is located near $\mu=1$ for small $\varepsilon$. The Krein signature, $\operatorname{sign}(K)$, of the internal mode for the Floquet multiplier $\mu=e^{i \Omega}$ in the upper half-circle is +1 if the potential $V$ is soft and -1 if $V$ is hard.

In general, independently of the expression for the potential $V$, the pair of phonon arcs on the unit circle (which represent Floquet multipliers for the wave continuum bands) does not overlap for small $\varepsilon$; one arc is located 
Morse potential: $\omega_{0}=0.85$

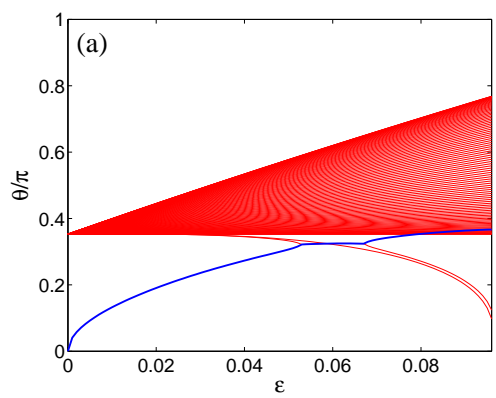

$\phi^{4}$ potential: $\omega=2$

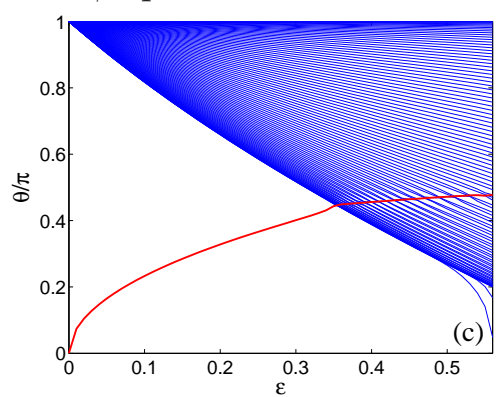

Morse potential: $\omega_{0}=0.65$

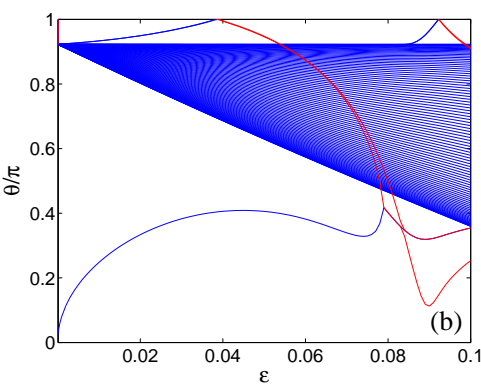

$\phi^{4}$ potential: $\omega_{0}=5$

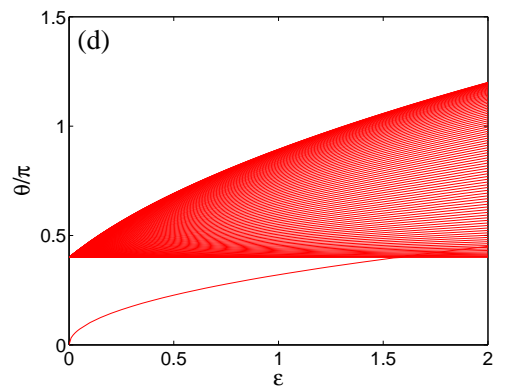

Figure 2. Floquet argument $\theta$ restricted on $[0, \pi]$ versus $\varepsilon$ for the twosite breathers oscillating in anti-phase for the Morse potential $(\mathrm{a}, \mathrm{b})$ and in phase for the $\phi^{4}$ potential (c,d). Red (blue) lines represent spectral modes with positive (negative) Krein signature.

in the upper half-circle and the other arc is located in the lower half-circle. The Krein signatures of the two distinct phonon arcs are opposite to each other. The arc in the upper half-circle has $\operatorname{sign}(K)=-1$ if $\pi<T<2 \pi$ or $3 \pi<T<4 \pi$ and $\operatorname{sign}(K)=1$ if $0<T<\pi$ or $2 \pi<T<3 \pi$, where $T$ is the breather period. Recall that $T<2 \pi$ for hard potentials and $T>2 \pi$ for soft potentials. For an earlier discussion of these spectral features, see e.g. [21, 22].

If $2 \Omega$ lies inside the wave continuum, the breathers are subject to the nonlinear instability mechanism presented herein, as long as the Krein signature of the phonon arc at the upper half-circle is the opposite of the Krein signature of the internal mode residing in this half-circle. This implies that for the configurations above, nonlinear instability can arise for the intervals $\pi<T<2 \pi$ (hard potentials) and $2 \pi<T<3 \pi$ (soft potentials). However, nonlinear instability cannot be observed in the intervals $0<T<\pi$ (hard potentials) and $3 \pi<T<4 \pi$ (soft potentials). All 
four cases are represented in Fig. 2, where the dependence of the relevant arguments $\theta$ of the Floquet multipliers $\mu=e^{i \theta}$ is displayed versus the coupling constant $\varepsilon$. For further experiments, we represent the unstable and stable cases as follows:

- Morse potential: unstable case $-\varepsilon=0.03$ for $\omega_{0}=0.85(T \approx 2.35 \pi)$; stable case $-\varepsilon=0.04$ for $\omega_{0}=0.65(T \approx 3.08 \pi)$.

- $\phi^{4}$ potential: unstable case $-\varepsilon=0.3$ for $\omega_{0}=2(T=\pi)$; stable case $-\varepsilon=1$ for $\omega_{0}=5(T=0.4 \pi)$.

In order to observe the emergence of the nonlinear instability, breathers are perturbed by adding the internal mode $(\mathbf{W}(0), \dot{\mathbf{W}}(0))$, multiplied by a relatively small factor $\delta$, to the breather solution $(\mathbf{u}(0), \dot{\mathbf{u}}(0))$. The reported numerical integration results have been obtained by means of the 4th order explicit and symplectic Runge-Kutta-Nyström method developed in [9, 32]. This scheme preserves the energy up to a $\mathcal{O}\left(10^{-8}\right)$ factor even for the long integration times $\left(\gtrsim 10^{4}\right)$ used here.

Fig. 3 shows the outcome for dynamics of the two-site anti-phase breather in the Morse potential for $\varepsilon=0.03$ for $\omega_{0}=0.85(T \approx 2.35 \pi)$. In this case, the internal mode eigenfrequency is $\Omega \approx 0.1002$, whereas the nearest phonon band shifted by $k_{0}=1$ [see (25) and (26)] is located in the frequency range $[0.15,0.2083]$. As a result, the double frequency $2 \Omega$ is inside the phonon band. The two-site breather is perturbed by the internal mode multiplied by $\delta=0.2$.

Panels (a) and (b) of Fig. 3 include the breather profile with the Floquet spectrum and the internal mode $(\mathbf{W}, \dot{\mathbf{W}})$ at $t=0$. Panel (c) shows the energy density at the central sites $n=0,1$. The energy density is defined by

$$
h_{n}=\frac{\dot{u}_{n}^{2}}{2}+V\left(u_{n}\right)+\frac{\varepsilon}{4}\left[\left(u_{n}-u_{n+1}\right)^{2}+\left(u_{n}-u_{n-1}\right)^{2}\right],
$$

so that the Hamiltonian in (44) can be written as $H=\sum_{n \in \mathbb{Z}} h_{n}$. Panel (d) compares the profile of the perturbed breather at $t=0$ to the profile of the breather near the end of the simulation.

From Fig. 3k, we observe the nonlinear instability of the two-site breather with frequency $\omega_{0}=0.85$, which manifests by a sudden decay of the energy density at the $n=1$ site and the growth of the energy density at the $n=0$ site. A quasi-periodic single-site breather is formed as a result of this instability of the two-site breather.

Fig. 4 shows the outcome for dynamics of the two-site in-phase breather in the hard $\phi^{4}$ potential for $\varepsilon=0.3, \delta=0.2$, and $\omega_{0}=2$ $(T=\pi)$. For this case, the internal mode eigenfrequency is $\Omega \approx 0.4011$, whereas the nearest phonon band shifted by $m_{0}=1$ [see (25) and (26)] is located in the frequency range $[0.56,2]$. Again, the double frequency $2 \Omega$ 

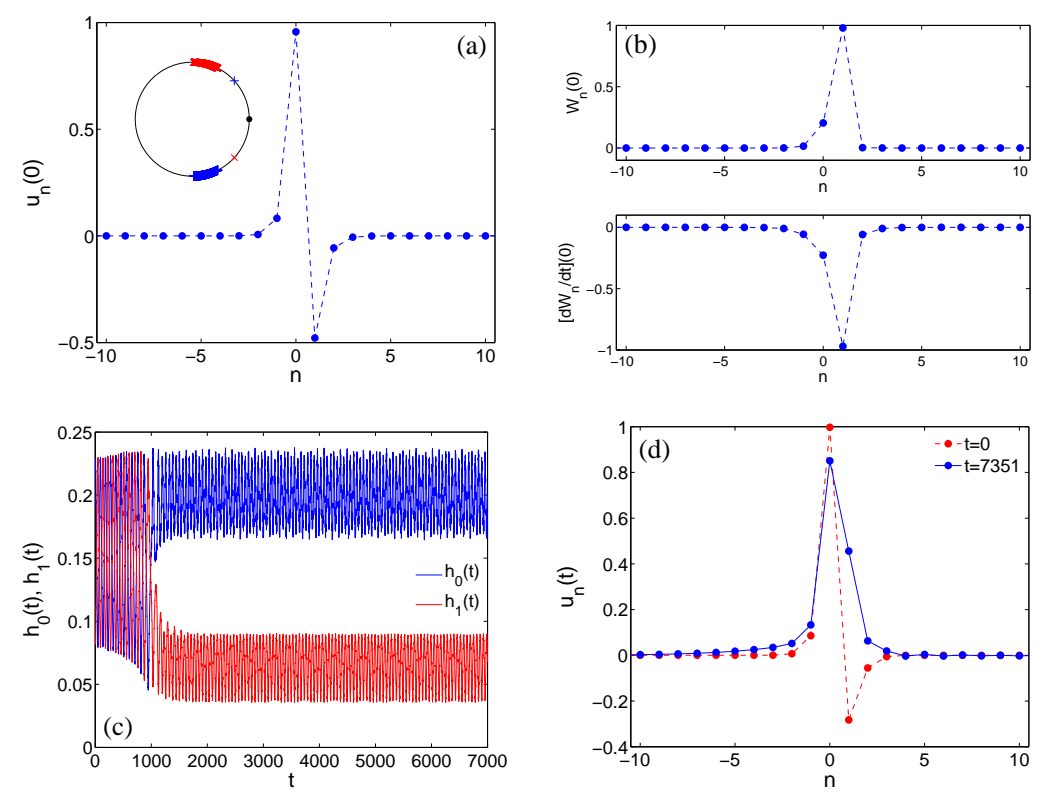

Figure 3. The two-site anti-phase breather, predicted to be nonlinearly unstable, in the Morse potential for $\varepsilon=0.03$ for $\omega_{0}=0.85$ : the stationary breather profile and Floquet spectrum (a), the internal mode (b), the energy density versus time (c), and the profile of the perturbed breather at $t=0$ and at the end of the simulation $(\mathrm{d})$. 

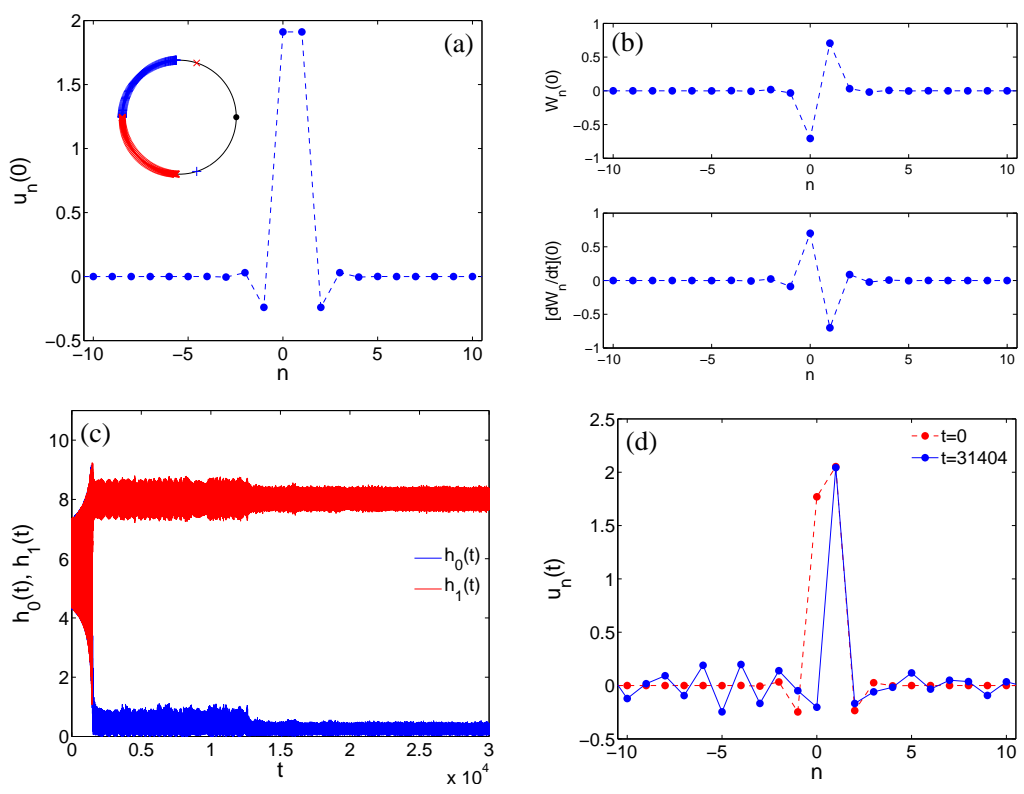

Figure 4. Similar to Fig. 3 but now for the two-site in-phase breather in the hard $\phi^{4}$ potential for $\varepsilon=0.3$ and $\omega_{0}=2$.

is inside the phonon band. As a result, similarly to Fig. 3, we observe the nonlinear instability of the two-site breather and its transformation to a quasi-periodic single-site breather.

We point out that the final state in both computations of Figs. 3 and 4 consists of quasi-periodic oscillations of a single-site breather. This behavior appears to be generic for the range $[\pi, 2 \pi]$ of the periods $T$ supported by the hard $\phi^{4}$ potential and the range $[2 \pi, 3 \pi]$ of the periods $T$ supported by the soft Morse potential.

Figs. 5 and 6 show the cases predicted to be nonlinearly stable in the dynamics of two-site breathers. For the soft Morse potential, we take the anti-phase breather for $\varepsilon=0.04$ and $\omega_{0}=0.65(T \approx 3.08 \pi)$, with the perturbation parameter $\delta=0.025$. The internal mode possesses the frequency $\Omega=0.1319$ and the nearest phonon band is shifted by $m_{0}=2$ to $[0.22,0.3]$, so that $2 \Omega$ is inside the phonon band. For the hard $\phi^{4}$ potential, we take the in-phase breather for $\varepsilon=1, \delta=0.2$, and $\omega_{0}=5$ $(T=0.4 \pi)$. The internal mode frequency is $\Omega=0.8013$ and the nearest phonon band is not shifted $\left(k_{0}=0\right)$ and is located at $[1,2.23]$, so that $2 \Omega$ is again inside the phonon band. In both cases, the internal mode and the phonon band in the upper half-circle have the same Krein signature. 
Despite performing long-time dynamical simulations, an instability of the two-site breathers does not seem to arise, confirming our theoretical predictions. Due to the Hamiltonian nature of the dynamics and the excitation of the initial perturbation, we observe a quasi-periodic dynamics of the original two-site breathers, however, there are fundamental differences observed between the oscillatory dynamics of Figs. [5] and [6 and the unstable ones involving growth (and the eventual destruction of the two-site breather states) of Figs. 3 and 4 .

\section{Conclusions and Future Challenges}

In the present work, we explored a broad question regarding the stability of multi-site breathers in Klein-Gordon lattices. This question arises in a wide array of settings where discrete breathers emerge, namely in nonlinear dynamical lattices. Following the earlier work on discrete (and continuous) nonlinear Schrödinger models [11, 15, we systematically developed the notion of the Krein signature for the wave continuum and the internal modes associated with the excited breather states. Subsequently, we produced asymptotic expansions that illustrated the occurrence of the nonlinear instability of multi-breathers, when (second) harmonics of internal modes resonate with the wave continuum of the opposite Krein signature.

However, we also revealed that contrary to the case of discrete (and continuous) nonlinear Schrödinger models, depending on the period of the breather, it is possible to identify parametric regimes for both soft and hard nonlinearities, where the multi-site breathers are nonlinearly stable. We have confirmed all of the above conclusions through direct numerical simulations of two on-site potentials, namely the soft Morse and the hard $\phi^{4}$ potentials.

This instability poses a number of interesting questions for future studies. On the one hand, it is relevant to explore theoretically whether generalizations of this mechanism can be numerically observed for higher-thansecond harmonics (with a suitably modified growth law). Furthermore, the mechanism is expected to persist not only for multi-site breather configurations bearing a larger number of sites, but also in higher dimensional setups and for structurally more complex excited state configurations, such as vortex breathers. On the other hand, the nonlinear nature of the instability and its weaker-than-exponential growth poses a substantial challenge towards its experimental realization. Finally, a deeper understanding of the evolution stages of the instability beyond its onset would be an important (albeit potentially formidable) task for the dynamics of the nonlinear models of interest. 

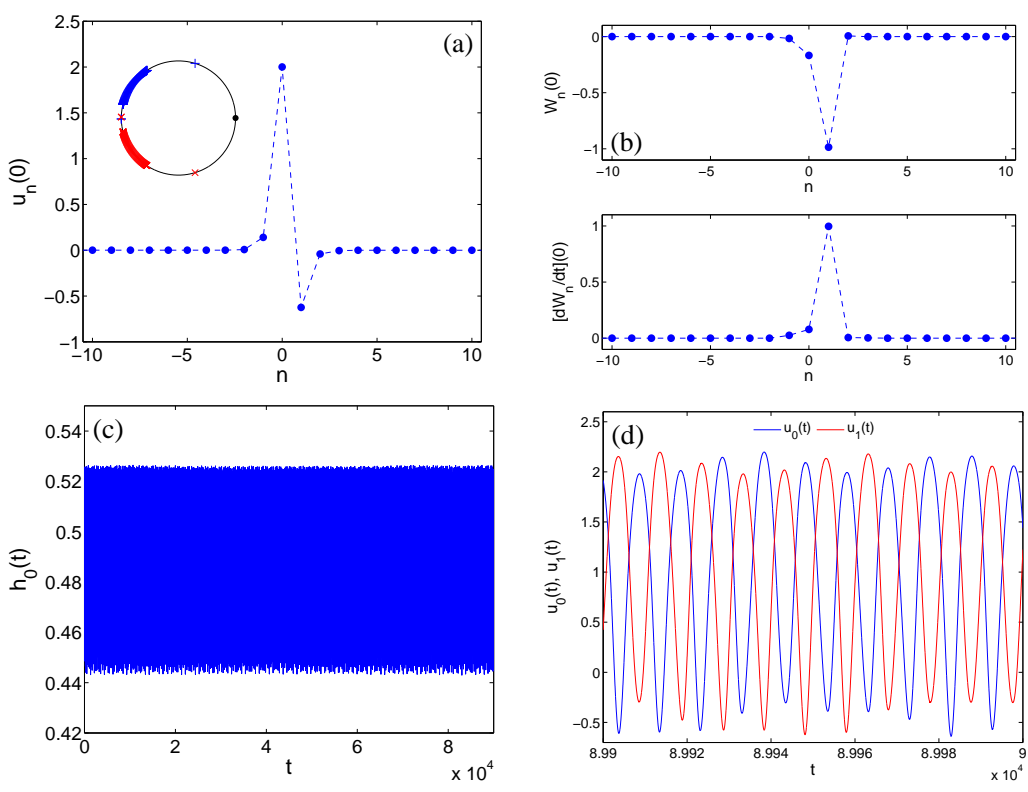

Figure 5. The two-site anti-phase breather, predicted to be nonlinearly stable, in the Morse potential for $\varepsilon=0.04$ for $\omega_{0}=0.65$ : the stationary breather profile and Floquet spectrum (a), the internal mode (b), and the energy density of the central site versus time (c). Panel (d) shows the evolution of the central sites $n=0$ and $n=1$. 

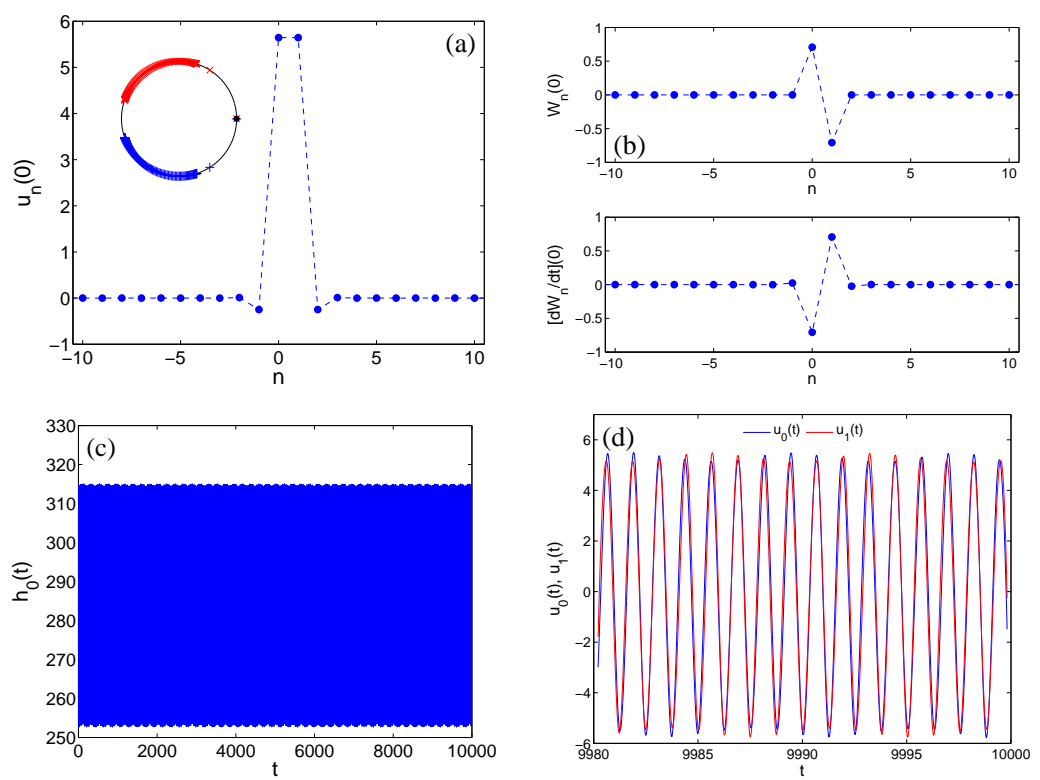

Figure 6. Similar to Fig. 3 but now for the two-site in-phase breather in the hard $\phi^{4}$ potential for $\varepsilon=1$ for $\omega_{0}=5$.

\section{Acknowledgments}

The authors are grateful to Dr. Avadh Saxena for seeding this collaboration and for valuable discussions. P.G.K. gratefully acknowledges the support of NSF-DMS-1312856, as well as from the US-AFOSR under grant FA9550-12-1-0332, and the ERC under FP7, Marie Curie Actions, People, International Research Staff Exchange Scheme (IRSES-605096). P.G.K.'s work at Los Alamos is supported in part by the U.S. Department of Energy. The work of D.P. is supported by the Ministry of Education and Science of Russian Federation (the base part of the state task No. $2014 / 133)$.

\section{References}

1. A. Achilleos, A. Álvarez, J. Cuevas, D.J. Frantzeskakis, N.I. Karachalios, P.G. Kevrekidis, B. Sanchez-Rey, Escape dynamics in the discrete repulsive $\phi^{4}$ model, Physica D 244:1-24 (2013).

2. J.F.R. Archilla, J. Cuevas, B. SÁnchez-Rey and A. Álvarez, Demonstration of the stability or instability of multibreathers at low coupling, Physica D 180:235255 (2003). 
3. S. Aubry, Breathers in nonlinear lattices: Existence, linear stability and quantization, Physica D 103:201-250 (1997).

4. D. Bambusi, Exponential stability of breathers in Hamiltonian networks of weakly coupled oscillators, Nonlinearity 9:433-457 (1996).

5. D. Bambusi, Asymptotic stability of breathers in some Hamiltonian networks of weakly coupled oscillators, Comm. Math. Phys. 324:515-547 (2013).

6. G. Bartal, O. Cohen, T. Schwartz, O. Manela, B. Fredman, M. Segev, H. BulJan and N.K. Efremidis, Spatial photonics in nonlinear waveguide arrays, Opt. Express 13:1780-1796 (2005).

7. M. Betti and D.E. Pelinovsky, Periodic travelling waves in dimer granular chains, J. Nonlinear Sci. 23:689-711 (2013).

8. N. Boechler, G. Theocharis, S. Job, P.G. Kevrekidis, M. Porter and C. Daraio, Discrete breathers in one-dimensional diatomic granular crystals, Phys. Rev. Lett. 104:244302 (2010).

9. M.P. Calvo and J.M. SAnz-Serna, High-order symplectic Runge-KuttaNystrom methods, SIAM J. Sci. Comput. 14:936-952 (1993).

10. C. Chong, F. Li, J. Yang, M.O. Williams, I.G. Kevrekidis, P.G. Kevrekidis and C. DARAio, Damped-driven granular chains: An ideal playground for dark breathers and multibreathers, Phys. Rev. E 89: 032924 (2014).

11. S. Cuccagna, On instability of excited states of the nonlinear Schrdinger equation, Physica D 238:38-54 (2009).

12. J. Cuevas, V. Koukouloyannis, P.G. Kevrekidis and J.F.R. Archilla, Multibreather and vortex breather stability in Klein-Gordon lattices: Equivalence between two different approaches, Int. J. Bif. \& Chaos 21:2161-2177 (2011).

13. S. Flach and C.R. Willis, Discrete breathers, Phys. Rep. 295:181-264 (1998).

14. S. Flach and A.V. Gorbach, Discrete breathers: Advances in theory and applications, Phys. Rep. 467:1-116 (2008).

15. P.G. Kevrekidis, D.E. Pelinovsky and A. Saxena, When linear stability does not exclude nonlinear instability, Phys. Rev. Lett. (2015), in press.

16. Yu.S. Kivshar, D.E. Pelinovsky, T. Cretegny and M. Peyrard, Internal modes of solitary waves, Phys. Rev. Lett. 80:5032-5035 (1998).

17. V. Koukouloyannis and P.G. Kevrekidis, On the stability of multibreathers in Klein-Gordon chains, Nonlinearity 22:2269-2285 (2009).

18. F. Lederer, G.I. Stegeman, D.N. Christodoulides, G. Assanto, M. Segev and Y. Silberberg, Discrete solitons in optics, Phys. Rep. 463:1-126 (2008), .

19. R. S. MacKay, Discrete breathers: classical and quantum, Physica A 288:174198 (2000).

20. R.S. MAcKAY and S. AubrY, Proof of existence of breathers for time-reversible or Hamiltonian networks of weakly coupled oscillators, Nonlinearity 7:1623-1643 (1994).

21. R.S. Mackay and J.-A. Sepulchre, Stability of discrete breathers, Physica D 119:148-162 (1998).

22. J.L. Marín, S. Aubry and L.M. Floría, Intrinsic localized modes: discrete breathers. existence and linear stability, Physica D 113:283-292 (1998).

23. O. Morsch and M. Oberthaler, Dynamics of Bose-Einstein condensates in optical lattices, Rev. Mod. Phys. 78:179-215 (2006).

24. J.B. PAge, Asymptotic solutions for localized vibrational modes in strongly anharmonic periodic systems, Phys. Rev. B 41:7835-7838 (1990).

25. D. Pelinovsky, T. Penati, and S. Paleari, Approximation of small-amplitude weakly coupled oscillators with discrete nonlinear Schrödinger equations, Rev. 
Math. Phys. (2015), submitted.

26. D.E. Pelinovsky, P.G. Kevrekidis and D.J. Frantzeskakis, Stability of discrete solitons in nonlinear Schrödinger lattices, Physica D 212:1-19 (2005).

27. D.E. Pelinovsky, Yu.S. Kivshar and V.V. Afanasjev, Internal modes of envelope solitons", Physica D 116:121-142 (1998).

28. D.E. Pelinovsky and A. SAKovich, Internal modes of discrete solitons near the anti-continuum limit of the dNLS equation, Physica D 240:265-281 (2011).

29. D.E. Pelinovsky and A. Sakovich, Multi-site breathers in Klein-Gordon lattices: stability, resonances, and bifurcations, Nonlinearity 25:3423-3451 (2012).

30. M. PeYrard, Nonlinear dynamics and statistical physics of DNA, Nonlinearity 17:R1-R40 (2004).

31. Z. RAPTI, Stationary solutions for a modified Peyrard-Bishop DNA model with up to third-neighbor interactions, European Journal of Physics E 32:209-216 (2010).

32. J.M. SAnZ-Serna and M.P. CALvo, Numerical Hamiltonian Problems (Chapman and Hall, London, 1994).

33. M. Sato, B.E. Hubbard and A.J. Sievers, Nonlinear energy localization and its manipulation in micromechanical oscillator arrays, Rev. Mod. Phys. 78:137-157 (2006).

34. A.J. Sievers and S. Takeno, Intrinsic localized modes in anharmonic crystals, Phys. Rev. Lett. 61:970-973 (1988).

Grupo de Física No Lineal, Departamento de Física Aplicada I, Universidad de Sevilla. Escuela Politécnica Superior, C/ Virgen de África, 7, 41011-Sevilla, SPAin;

Instituto de Matemáticas de la Universidad de Sevilla (IMUS). Edificio

Celestino Mutis. Avda. Reina Mercedes s/n, 41012-Sevilla, Spain

Department of Mathematics and Statistics, University of Massachusetts, AMHERST, MA 01003-9305, USA;

Center for Nonlinear Studies and Theoretical Division, Los Alamos

National Laboratory, Los Alamos, New Mexico 87545, USA

Department of Mathematics, McMaster University, Hamilton, Ontario, CANADA, L8S 4K1;

Department of Applied Mathematics, Nizhny Novgorod State Technical University, Nizhny Novgorod, Russia 